\title{
DESEMPENHO DE UM SISTEMA DE IRRIGAÇÃO POR GOTEJAMENTO EM CANA-DE-AÇÚCAR
}

\author{
Renato Américo Araújo Neto*1, Pedro Luã Sarmento", Ricardo Araújo Ferreira Júnior ${ }^{1}$, Mauricio Bruno Prado \\ Silva ${ }^{1}$, Adolpho Emanuel Quintela Rocha ${ }^{1}$, lêdo Teodoro ${ }^{1}$
}

${ }^{1}$ Centro de Ciências Agrárias, Universidade Federal de Alagoas, BR 104 Norte, Km 85, Rio Largo, AL;

*Autor para correspondência: renato.eng.agronomo@hotmail.com

\begin{abstract}
RESUMO: $O$ trabalho objetivou avaliar a uniformidade e a eficiência de aplicação de água em um sistema de irrigação por gotejamento, na região de Rio Largo - AL, Brasil. 0 estudo foi realizado em um cultivo de cana-deaçúcar, com fitas gotejadoras, com emissores não autocompensantes e vazão nominal de 1,5 L h'-1. Em campo, selecionaram-se quatro linhas laterais e, em cada uma destas, oito emissores (primeiro, 1/7, 2/7, 3/7, 4/7, 5/7, $6 / 7$ e o último), totalizando 32 emissores. Após medidas as vazões de cada emissor, obteve-se o cálculo dos coeficientes de uniformidade, classificando-se o sistema quanto a sua eficiência de distribuição. 0 projeto foi considerado excelente, em relação à uniformidade de distribuição, com CUC e CUD apresentando os valores $96,29 \%$ e $94,32 \%$, respectivamente e as vazões encontram-se abaixo da vazão nominal.
\end{abstract}

PALAVRAS-CHAVE: coeficiente de uniformidade, gotejamento, métodos de uniformidade

\section{PERFORMANCE OF A DRIP IRRIGATION SYSTEM IN SUGARCANE}

ABSTRACT: The study aimed to evaluate the uniformity and efficiency of application of water in a drip irrigation system in the region of Rio Largo - AL, Brazil. The study was performed in a sugarcane crop, with drip lines with non-pressure compensating emitters and nominal flow rate of $1.5 \mathrm{~L} \mathrm{~h}^{-1}$. In the design, we selected four side lines, and each of the eight emitters (first, 1/7, 2/7, 3/7, 4/7, 5/7,6/7 and the last), a total of 32 emitters. After measures the flow rates of each emitter, we obtained the uniformity coefficient calculation, classifying the system as its distribution efficiency. The project was considered excellent, compared to the uniformity distribution, with CUC and CUD with values $96.29 \%$ and $94.32 \%$ respectively. The flow rates are below the nominal flow.

KEYWORDS: uniformity coefficient, drip irrigation, uniformities methods

\section{INTRODUÇÃO}

Algumas regiões brasileiras sofrem com a escassez de água para irrigação, que torna limitante a expansão da área irrigada, fazendo-se necessária implementação de práticas que reduzam o consumo excessivo de água na agricultura, dependente de uma estimativa adequada da evapotranspiração e de adoção de sistemas de irrigação que aumentem a eficiência de utilização da água, já que, as aplicações excessivas resultam em prejuízos consideráveis para as plantas e solo, com uma menor eficiência do uso de água para irrigação (Reis et al., 2005).

Dentre os sistemas de irrigação conhecidos, destaca-se a irrigação por gotejamento, devido a sua maior capacidade de eficiência do uso da água na agricultura, aplicando-se a lâmina somente na região da zona radicular das culturas, em pequenas intensidades, com alta frequência de aplicação (turno de rega chegando a quatro dias), mantendo-se a umidade do solo em torno da capacidade de campo (Bernardo, 2006).

Para que a irrigação torne-se mais eficiente é necessário que os sistemas apresentem um alto índice de uniformidade de aplicação da água, já que, uma vez implantado o projeto de irrigação, seguindo as recomendações do fabricante, é preciso confirmar as características do sistema em campo, avaliandose vazão, uniformidade de distribuição de água e eficiência de irrigação. Entretanto, tal metodologia ainda é utilizada por poucos agricultores, mesmo com 
acesso à tecnologia, muitos ainda irrigam de forma inadequada, por falta de orientação e conhecimento (Silva e Silva, 2005).

A uniformidade de aplicação de água, em sistemas de irrigação por gotejamento, pode ser expressa por vários coeficientes, destacando-se 0 coeficiente de uniformidade de Christiansen (CUC) e o coeficiente de uniformidade de distribuição (CUD) (Bernardo, 2006; Denículi et al., 1980; Keller e Karmelli, 1975), sendo mais frequente o uso do coeficiente de uniformidade de distribuição, já que este possibilita uma medida mais restrita, dando maior peso às plantas que receberam menos água (Lopez et al., 1992).

Para tanto, o presente trabalho teve como objetivo avaliar a uniformidade e a eficiência de aplicação de água em um sistema de irrigação por gotejamento instalado em um cultivo de cana-deaçúcar, na região de Rio Largo, Alagoas.

\section{MATERIAL E MÉTODOS}

A área de estudo foi no Centro de Ciências Agrárias (CECA), da Universidade Federal de Alagoas (UFAL), Rio Largo, Alagoas (09²8'02"S; 3549'43"W; $127 \mathrm{~m}$ ), em um cultivo de cana-de-açúcar (Saccharum spp.), variedade RB98710, em uma área de 240 $\mathrm{m}^{2}$, com 12 linhas de 20 metros de comprimentos espaçadas de $1,0 \mathrm{~m}$.

O sistema de irrigação avaliado foi do tipo gotejamento superficial (não autocompensante), com fitas gotejadoras de $16 \mathrm{~mm}$ de diâmetro. As fitas foram distribuídas nas linhas de plantio. Os emissores das fitas foram espaçados a cada 0,5 metros. Segundo especificações do fabricante os emissores sob uma pressão de $1,0 \mathrm{kgf}$. $\mathrm{cm}^{-2}$ proporcionam uma vazão nominal de $1,5 \mathrm{~L} \mathrm{~h}^{-1}$. No de avaliação as fitas gotejadoras têm 109 dias de uso. Para o adequado funcionamento do sistema foram instalados um filtro de disco e uma válvula reguladora de pressão para 1,0 kgf. $\mathrm{cm}^{-2}$.

A determinação da uniformidade de distribuição de água para o projeto, baseou-se na metodologia de Keller e Karmeli (1975), modificada por Denículi et al. (1980), em que são avaliadas 4 linhas laterais (a primeira linha, as localizadas a 1/3 e 2/3 da linha inicial e a última linha). Em cada linha foram avaliados oito emissores, localizados no início da fita gotejadora, 1/7, 2/7, 3/7, 4/7, 5/7, 6/7 e último gotejador, totalizando 32 gotejadores avaliados, utilizando-se de cronômetro, coletores e uma proveta para a obtenção dos dados. Com valores coletados foram calculados índices de uniformidades (CUC e CUD), Eficiência de Aplicação (EA) e Uniformidade Estatística (UE) de acordo com as equações 1 a 4 , respectivamente.

$$
C U C=100\left[1-\frac{\sum_{i}^{n}\left|q_{i}-\bar{q}\right|}{n \bar{q}}\right]
$$

em que:

$\mathrm{q}_{\mathrm{i}}=$ vazão de cada emissor, $\mathrm{L} \mathrm{h}^{-1}$;

$\bar{q}=$ vazão média dos emissores, $\mathrm{L} \mathrm{h}^{-1} ; \mathrm{e}$

$\mathrm{n}=$ número de emissores.

$C U D=100 \cdot\left(\frac{q_{n}}{\bar{q}}\right)$

em que:

$q_{n}=$ média de $25 \%$ das vazões com menores valores;

R $=K . C U D$

em que:

$\mathrm{Ks}=$ coeficiente de transmissividade. Para esse trabalho, utilizou-se o valor de $90 \%$, onde o Ks desejável está em torno de 85 a $90 \%$, segundo Vermeiren e Jobling, (1997).

$E=100\left(1-\frac{p}{\bar{q}}\right)$

em que:

$D p=$ desvio padrão

A interpretação dos valores de CUD baseou-se de acordo com a metodologia desenvolvida por Merriam e Keller (1978), com apresentação dos critérios, onde: $90 \%$, excelente; entre $80 \%$ e $90 \%$, bom; $70 \%$ e $80 \%$, regular; e menor que $70 \%$, ruim.

\section{RESULTADOS E DISCUSSÃO}

No quadro 1, apresentam-se os valores dos coeficientes de Uniformidade de Christiansen (CUC), de Uniformidade de Distribuição (CUD), Eficiência de 
Aplicação (EA), para o sistema de irrigação avaliado. Observou-se que o CUD $(96,29 \%)$ foi menor que 0 CUC $(94,32 \%)$ e, de acordo com Lopez et al. (1992), essa ocorrência foi devido ao fato do CUD ser um tratamento mais rigoroso quando relacionado a problemas na distribuição ao longo da linha lateral. Quando comparado com um sistema de aspersão, o gotejamento superficial apresenta-se com melhor porcentagem, onde, Santos et al. (2013) apresentou um CUC de aproximadamente $68 \%$. Souza et al. (2008) apresentou um CUC semelhante $(93,7 \%)$ ao avaliar um sistema de gotejamento na região nordeste do Paraná.
O sistema apresentou uma uniformidade de distribuição de 94,32\% que, de acordo com a metodologia descrita por Merriam e Keller (1978), esse valor é classificado como excelente (> 90\%). Com relação à uniformidade estatística, observa-se valores excelentes (95,13\%), de acordo com a classificação proposta por Braltz e Kesner (1983), citado por Souza et al. (2006). A Eficiência de aplicação apresentou aproximadamente $85 \%$ de eficiência (Tabela 1). Esse valor é contestado por Souza et al. (2008), que avaliando sistemas de irrigação, indicou uma eficiência de $100 \%$.

Quadro 1. Valores dos Coeficientes de Uniformidade de Christiansen (CUC) e o Coeficiente de Uniformidade de Distribuição (CUD), Eficiência de Aplicação (EA), para o projeto avaliado e a classificação do mesmo de acordo com Merriam e Keller (1978).

\begin{tabular}{cccccc}
\hline Sistema & CUC(\%) & CUD(\%) & EA(\%) & US(\%) & Classificação \\
\hline Gotejamento & 96,29 & 94,32 & 84,89 & 95,13 & Excelente \\
\hline
\end{tabular}

$\mathrm{Na}$ Figura 1, estão plotadas as vazões dos gotejadores distribuídos ao longo de cada linha lateral avaliada (inicial, 1/3, 2/3, final). Observou-se que a vazão média encontrava-se abaixo à vazão de projeto (especificada pelo fabricante), devido aos gotejadores não serem autocompensantes. Tem-se que, a vazão inicial do sistema encontra-se com valores abaixo da vazão média, fato também observado na vazão $1 / 3$ do sistema, que sofre uma forte queda na sua vazão. Essa diferença pode ser explicada pela existência de gotejadores com obstrução, devido a sólidos em suspensão, como exemplo, partículas de areia e silte podendo ser conduzidas para o interior do sistema, ou a presença de bolsas de ar na parte final das linhas laterais (Bonomo, 1999; Westcot, 1999, citado por Reis et al., 2005).

Figura 1. Vazões dos gotejadores distribuídos ao longo de cada linha lateral avaliada (inicial, 1/3, 2/3, final).
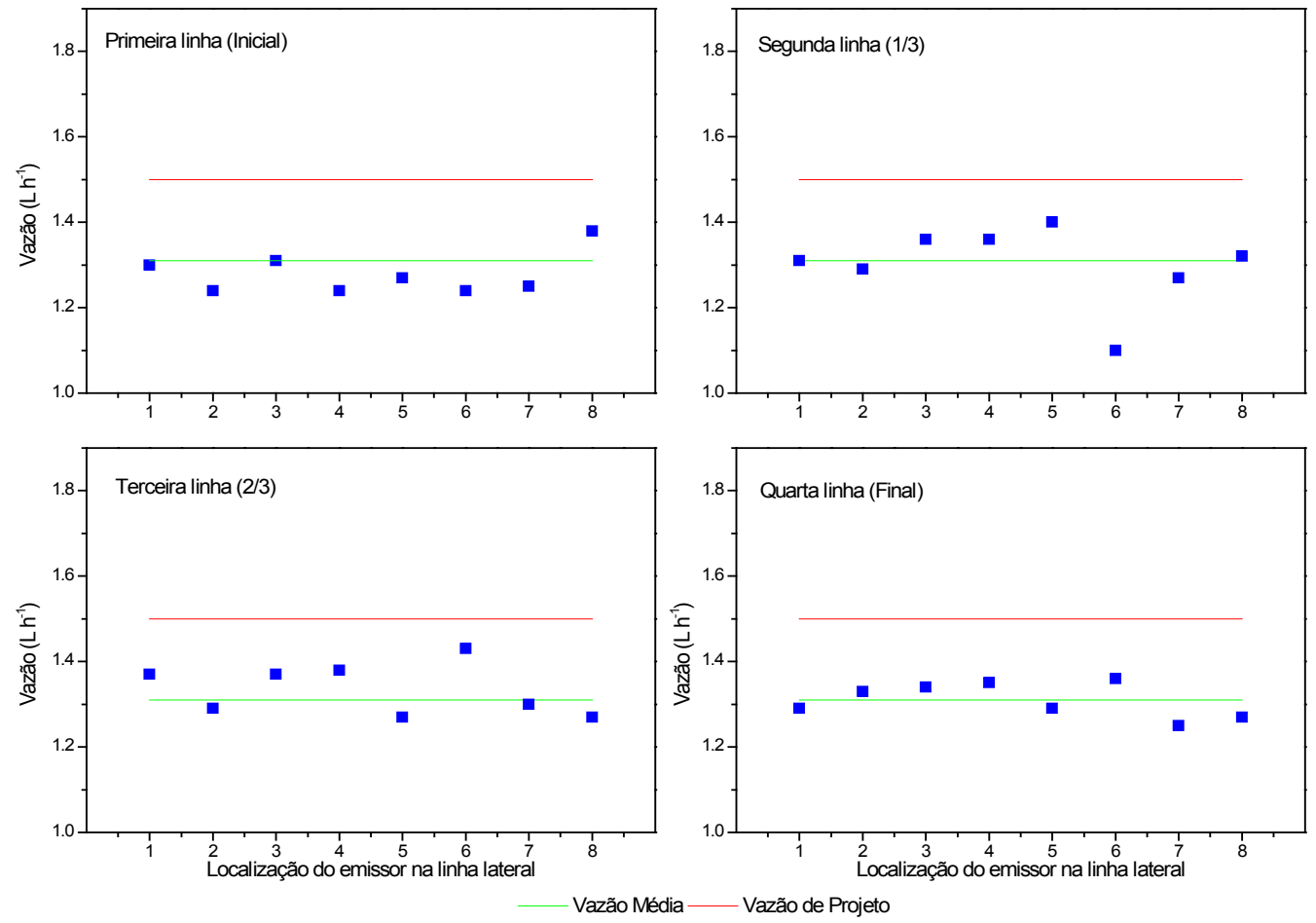
O sistema apresentou coeficiente de uniformidade superior ao recomendado para sistemas de irrigação localizada, que é de $90 \%$. A vazão real aplicada em cada linha estudada foi inferior à vazão de projeto.

\section{AGRADECIMENTOS}

À UFAL, pelo apoio institucional; ao CNPq e FAPEAL pelo apoio financeiro.

\section{REFERÊNCIAS BIBLIOGRÁFICAS}

AYERS, R. S.; WESTCOT, D. W. TRADUÇÃO DE GHEYI H. R., MEDEIROS J. F., DAMASCENO F. A. V., A qualidade da água na agricultura. (Estudos FAO: irrigação e Drenagem, 29). Campina Grande, Dept. Eng. Agrícola, UFPB, 1999.153p.

BERNARDO, S.; SOARES, A. A.; MANTOVANI, E. C. Manual de Irrigação. $8^{\mathrm{a}}$ ed. atu. e ampli. Viçosa: UFV, imprensa universitária, 2008, 625 p.

BONOMO, R. Análise da irrigação na cafeicultura em áreas de cerrado de Minas Gerais. Viçosa: UFV, 1999. 224p. Tese (Doutorado em Engenharia Agrícola) Universidade Federal de Viçosa.

BRALTS, V. F.; KESNER, C. D. Drip irrigation field uniformity estimation. Transactions of the ASAE, 1983, $26,5,1369-1374$.

DENÍCULI, W.; BERNARDO, S.; THIÁBAUT, J. T. L.; SEDIYAMA, G. C. Uniformidade de distribuição de água, em condições de campo num sistema de irrigação por gotejamento. Revista Ceres, 1980, 27, 50, 155-162.

KELLER, J.; KARMELI, D. Trickle irrigation design. Glendora: Rain Bird Sprinklers Manufacturing Corp., 1975. 133p.
LOPEZ, J. R.; ABREU, J. M. H.; REGALADO, A. P.; HERNANDEZ, J. F. G. Riego localizado. 2ed. Madrid: Centro Nacional de Tecnologia de Regadios, 1992. 217-229.

MERRIAM, J. L.; KELLER, J. Farm irrigation system evaluation: a guide for management. Logan: Utah State University, 1978. 271p.

REIS, E. F.; BARROS, F. M.; CAMPANHARO, M.; PEZZOPANE, J. E. M. Avaliação do desempenho de sistemas de irrigação por gotejamento. Engenharia na agricultura, 2005, 13, 2, 74-81.

SANTOS, M. A. L.; SILVA, S. DANTAS NETO, J.; LIMA, R. A. S.; RIBEIRO, P. H. P. Avaliação por canhão aspersos com uso de motobomba e eletrobomba. Enciclopédia Biosfera, 2013, 9, 16, 367-375.

SILVA, C. A.; SILVA, C. J. Avaliação de uniformidade em sistemas de irrigação localizada. R. Ci en. Eletrônica de Agronomia, 2005, Ano IV, 08.

SOUZA, E. A. M; SOUZA, P. C.; VILLAS BOAS, M. A. Avaliação do desempenho de sistemas de irrigação por aspersão convencional fixo e gotejamento em vila rural. Irriga, 2008, 13, 1, 47-62.

SOUZA, L. O. C.; MANTOVANI, E. C.; SOARES, A. A.; RAMOS, M. M.; FREITAS P. S. L. Avaliação de sistemas de irrigação por gotejamento, utilizados na cafeicultura. Revista Brasileira de Engenharia Ambiental, 2006, 10, 3, 541-548.

VERMEIREN, L.; JOBLING. G.A. Irrigação Localizada. Tradução de GHEY, H.R.; Damasceno, F.A.V.; Silva Júnior, L.G.A.; Medeiros, J.F. de. Campina Grande. UFPB. 1997. 184 p. (Estudos FAO: Irrigação e Drenagem, 36). 